\title{
Atividade larvicida do óleo de Anacardium bumile Saint Hill sobre Aedes aegypti (Linnaeus, 1762) (Diptera, Culicidae)
}

\author{
Larvicidal activity of Anacardium bumile Saint Hill oil \\ on Aedes aegypti (Linnaeus, 1762) (Diptera, Culicidae)
}

\author{
Karla Rejane de Andrade Porto ${ }^{1}$, Antonia Railda Roel², Márcia Marlene da Silva ${ }^{3}$, \\ Rosemary Matias Coelho ${ }^{4}$, Eloty Justina Dias Scheleder ${ }^{4}$ e Alex Haroldo Jeller ${ }^{5}$
}

\begin{abstract}
RESUMO
O objetivo deste estudo foi avaliar o potencial do cajuzinho do cerrado (Anacardium humile) sobre larvas de Aedes aegypti. Os extratos hexânico, etanólico, aquoso e o óleo das folhas foram obtidos do material vegetal coletado em fragmento de cerrado. Estes foram testados nas concentrações $1 \% ; 0,5 \%, 0,25 \%, 0,125 \%, 0,05 \%$ e $0,0125 \%$ diluídas em dimetil sulfóxido $1 \%$. A contagem das larvas mortas foi realizada após 24 horas. Utilizouse o método Probit de análise para obtenção das $\mathrm{CL}_{50}$ e respectivos intervalos de confiança. Conclui-se que apenas o óleo extraído de folhas de Anacardium humile causa $100 \%$ de mortalidade em larvas de $4^{\circ}$ estádio de Aedes aegypti nas concentrações até 0,125\%, o que parece indicar que os ingredientes ativos estão na fase mais apolar. 0 que indica a potencialidade de uso da planta como larvicida de Aedes aegypti, entretanto, novos testes deverão ser conduzidos utilizando outros órgãos vegetais, assim como outros métodos e solventes utilizados na extração.
\end{abstract}

Palavras-chaves: Inseticidas botânicos. Toxicicidade. Cajuzinho do cerrado.

\begin{abstract}
The objective of this study was to evaluate the potential of Anacardium humile (monkey nuts) against Aedes aegypti larvae. Hexane, ethanol and aqueous extracts and oil from leaves were obtained from plant material collected from the Brazilian savanna. These were tested at concentrations of $1 \%, 0.5 \%, 0.25 \%, 0.125 \%, 0.05 \%$ and $0.0125 \%$, diluted in $1 \%$ dimethyl sulfoxide. The dead larvae were counted 24 hours later. The Probit analysis method was used to obtain the $\mathrm{LC}_{50}$ and the respective confidence intervals. The conclusion was that only the oil extracted from Anacardium humile leaves caused $100 \%$ mortality among fourth-instar Aedes aegypti larvae, using concentrations of up to $0.125 \%$. This seems to indicate that the active ingredients are present in the most apolar phase. This indicates that this plant has potential use as a larvicide against Aedes aegypti. However, new tests should be carried out using other plant organs, as well as using other methods and solvents for the extraction.
\end{abstract}

Key-words: Botanical insecticides. Toxicity. Monkey nut.

A crescente urbanização proporcionou condições favoráveis para a propagação do Aedes aegypti, especialmente pelo uso intensivo de materiais descartáveis aumentando, assim, o número de criadouros potenciais para o mosquito vetor do flavivírus causador da dengue. 0 mesmo dissemina-se conforme a expansão doAedes aegypti, que hoje ocupa praticamente toda a faixa cosmo tropical da terra. As epidemias, desencadeadas a partir de 1986 no Brasil, estão sempre relacionadas com a introdução de um novo sorotipo do vírus ${ }^{1}$.

Para o controle de epidemias da dengue, paralelamente a campanhas educacionais junto à população, são necessárias pulverizações com inseticidas na região peridomiciliar. 0 uso de inseticidas químicos sintéticos tem sido a primeira opção para 0 controle dos adultos ou as formas larvais do Aedes aegypti nas campanhas nacionais. A utilização freqüente em grande escala destes produtos tem contribuído para o surgimento de problemas significativos como o aparecimento de resistência. Dessa forma, a busca de novas substâncias, principalmente aquelas extraídas de plantas, tem recebido especial atenção como forma de controle desse inseto ${ }^{1718}$.

As falhas operacionais de campanhas no controle do Aedes aegypti e o aparecimento de populações resistentes aos inseticidas utilizados revelam a necessidade de mudanças nas ações de controle e no manejo dos produtos. Esta situação é

\footnotetext{
1. Curso de Nutrição, Universidade Católica Dom Bosco, Campo Grande, MS. 2. Mestrado em Biotecnologia, Universidade Católica Dom Bosco, Campo Grande, MS. 3. Acadêmica do Curso de Nutrição - PIBIC/CPP - Universidade Católica Dom Bosco, Campo Grande, MS. 4. Curso de Farmácia, Universidade para o Desenvolvimento do Estado e da Região do Pantanal, Anhanguera Educacional, Campo Grande, MS. 5. Curso de Química, Universidade Estadual de Mato Grosso do Sul, Dourados, MS. Apoio Financeiro: Ministério de Ciência e Tecnologia, MCT por meio do Centro de Pesquisas do Pantanal - CPP

Endereço para correspondência: Dra. Antonia Railda Roel. Universidade Católica Dom Bosco. Av. Tamandaré 6000, Jardim Seminário, 79117-900 Campo Grande, MS e-mail: mbiotec@ucdb.br

Recebido para publicação em 14/07/2008

Aceito em 12/11/2008
} 
relatada como causa de resistência em vários locais como Cuba, Caribe, Venezuela, Ilhas Virgens e no Brasil, em São Paulo, Mato Grosso do Sul, Distrito Federal, Rio de Janeiro, Sergipe, Alagoas e Ceará ${ }^{12}$.

Na busca por controle químico alternativo contra Aedes aegypti, muitas pesquisas são desenvolvidas no intuito de se descobrirem novas substâncias inseticidas de origem vegetal. Acredita-se que a presença de biomoléculas em determinadas espécie vegetais sirvam como fator de seleção e adaptação evolucionária, que devem ser avaliadas quanto ao seu potencial de ação e toxicidade ${ }^{2}$. 0 potencial dos fitoterápicos proporciona a utilização de biomoléculas com atividade específica no controle de vetores de doenças endêmicas que, pela sua complexidade de composição, diminuem os riscos de resistência e minimiza a toxicidade para o meio ambiente.

Óleos essenciais de dez espécies vegetais, diluídos em solução aquosa de dimetil sufóxido nas concentrações de 100 , 50,10 e $1 \mathrm{mg} / \mathrm{mL}$, foram testados quanto sua atividade larvicida. Concluiu-se que o óleo essencial de Vanillosmopsis arborea induziu a maior atividade larvicida, com $\mathrm{CL}_{50}$ de $15,9 \mathrm{mg} / \mathrm{mL}$ e $\mathrm{CL}_{90}$ de $28,5 \mathrm{mg} / \mathrm{mL}$, enquanto o de Ocimum gratissimum $\mathrm{L}$. apresentou a menor atividade com $\mathrm{CL}_{50}$ de $95,80 \mathrm{mg} / \mathrm{mL}$ e $\mathrm{CL}_{90}$ de $102,86 \mathrm{mg} / \mathrm{mL}^{8}$.

Estudos realizados com plantas da região nordeste do Brasil, também apresentaram elevada ação larvicida ao Aedes aegypti. Os resultados mostraram que tanto o Ocimum americanum, como o Ocimum gratissimum apresentaram $\mathrm{CL}_{50}$ de $67 \mathrm{ppm}$ e de 60ppm, respectivamente, comparado aos obtidos com Lippia sidoides que têm $\mathrm{CL}_{50}$ a 63ppm e o Cymbopogon citratus a 69ppm, ambos já utilizados em escala comercial. Estes resultados sugerem a ação potencial do óleo essencial destas espécies de Ocimum para o controle do Aedes aegyptit.

Estudos químicos de várias espécies do gênero Croton têm mostrado que os compostos isolados apresentam elevada atividade larvicida sobre diferentes espécies de mosquitos, incluindo 0 Aedes aegypti. Dentre as diferentes espécies estudadas, com atividade comprovada, destacam os extratos do caule e da folha do Croton argyrophylloides, Croton nepetaefolius, Croton sonderianus, e Croton zehntneri. Óleos de Croton argyrophylloides e Croton sonderianus também causou 100\% de mortalidade das larvas. Este estudo mostrou que todos os órgãos das espécies vegetais analisadas apresentaram propriedades larvicidas ${ }^{4}$.

A família Anacardiaceae, em especial o gênero Anacardium, destaca-se pelo número de investigações relativas à composição química de suas espécies, atividades biológicas de seus extratos e metabólitos principalmente pela presença dos compostos fenólicos e catecólicos, ou à mistura destas substâncias, denominados lipídios fenólicos ${ }^{616}$.

Estudos realizados demonstram que os lipídios fenólicos são encontrados nas espécies de Anacardiaceae e normalmente apresentam propriedades tóxicas ou alergênicas ${ }^{6}$. Os lipídios fenólicos, derivados da rota policetídica, metabólitos freqüentes em espécies desta família, possuem muitas atividades biológicas comprovadas. Entre os lipídios fenólicos, os ácidos anacárdicos juntamente com os cardois são os principais constituintes estudados quanto à atividade biológica e em especial os ácidos anacárdicos obtidos de Anacardium occidentale mostraram-se larvicida na concentração de 10ppm contra Aedes fluviatilis (Lutz) ${ }^{5}$.

Além dos lipídios fenólicos, entre os compostos lipofílicos presentes em óleos essenciais, destacam-se os terpenos, classe esta de metabólitos secundários apontada com atividade para Aedes aegypti. Foi relatado, também, que os óleos essenciais contendo sesquiterpenos como nerolidol e farnesol, monoterpenos como $\alpha$-pireno, carvona, geraniol e, ainda os fenilpropanóides como safrol, eugenol e aldeídos cinâmicos, apresentaram atividade biocida em larvas de Aedes aegypti $i^{17}$.

Entre as espécies deste gênero, além da Anacardium occidentale, destaca-se a Anacardium bumile, conhecida como cajuzinho-do-cerrado ou cajuí, nativa do Brasil e de ocorrência natural em campo sujo, no cerrado e no pantanal sul Matogrossense $\mathrm{e}^{314}$. Estudos têm sido realizados para descobrir as propriedades e benefícios da Anacardium bumile. Nos últimos 30 anos, alguns estudos demonstraram que esta possui atividade antifúngica, anti-rotavirus, antidiarréica, antiinflamatória, hipoglicemiante ${ }^{910} 15$.

Considerando a diversidade de espécies da família Anacardiaceae em Mato Grosso do Sul, em especial do gênero Anacardium $^{5613}$, e a potencialidade dos lipídios fenólicos, e em se tratando da Anacardium bumile ${ }^{6814}$, não há informações sobre estudos efetuados com extratos e óleo obtidos das folhas. E, considerando ainda, a alta infestação do vetor da dengue e os surtos de dengue na região Centro-oeste, reconhecida como uma zona de alta prevalência, objetivou-se avaliar o potencial inseticida do óleo das folhas de Anacardium humile sobre as larvas Aedes aegypti.

\section{MATERIAL E MÉTODOS}

O material vegetal, folhas de Anacardium bumile, foi coletado entre julho e agosto de 2006, em um fragmento de cerrado, localizado dentro do Campus III da Universidade para o Desenvolvimento do Estado e da Região do Pantanal (UNIDERP), Campo Grande-MS. As amostras botânicas foram identificadas pela botânica Prof ${ }^{a}$ MSc Eloty Justina Dias Schleder e as exsicatas foram depositadas no Herbário da UNIDERP sob os registros: $n^{0} 5558$ (coleta 08/08/2006) e 6517 (coleta dia 20/10/2006).

As folhas frescas $(450 \mathrm{~g})$ foram submetidas à trituração em liquidificador doméstico. Em sequiência para extração do óleo essencial, o material processado foi submetido à técnica tradicional de arraste com vapor d'água, por cinco horas através do aparelho de Clevenger obtendo-se 0,5mL/100g.

As folhas secas, em estufa de circulação de ar $\left(40^{\circ} \mathrm{C}\right)$ de Anacardium bumile (500g), foram extraídas seqüencialmente com solventes de polaridade crescente, n-hexano, clorofórmio, etanol e água em aparelho extrator Soxhlet, por quatro horas, utilizando o resíduo da primeira extração para retomar a próxima extração. Os solventes foram evaporados em rotaevaporador e das frações obtidas, foram utilizadas nos bioensaios ${ }^{10}$ apenas as frações: hexânica, etanólica e aquosa. 
Os bioensaios foram executados no Laboratório de Entomologia da Universidade Católica Dom Bosco. As larvas de Aedes aegypti foram fornecidas pela criação estoque, seguindo a metodologia de colonizaçãa $0^{5}$. Em todas as etapas, foram utilizadas larvas do $4^{\circ}$ estádio num total de 25 indivíduos para cada $25 \mathrm{ml}$ de solução, em recipiente de vidro de $200 \mathrm{ml}$. Foram utilizadas as concentrações de $1 \% ; 0,5 \%, 0,25 \% ; 0,125 \%$; $0,05 \%$ e $0,0125 \%$ do óleo e dos extratos das folhas, diluídas em DMSO a 1\% (v/v), em quadruplicata pelo período de 24 horas. Paralelamente, foi conduzido o controle negativo (branco) e o positivo (Rotenona).

Utilizou-se o método análise Probit, por meio do programa POLO-PC, para obtenção das $\mathrm{CL}_{50}$ e respectivos intervalos de confiança. Após a avaliação da toxicidade de todas as concentrações, os testes foram refeitos para confirmar os dados obtidos por mais duas vezes consecutivas, e só então calculada a concentração letal (CL).

\section{RESULTADOS}

Não foi constatado efeito tóxico das frações hexânica, etanólica e aquosa obtidas das folhas de Anacardium bumile sobre as larvas de Aedes aegypti.

Entretanto, o óleo retirado das folhas causou efeito tóxico para as larvas. De acordo com análise por Probit, definiu-se a $\mathrm{CL}_{50}$ na concentração de 20,9ppm, próximo a concentração de $0,025 \%$ o que torna o produto um potencial larvicida, porém de elevada toxicidade. A concentração mínima capaz de produzir mortalidade $\left(\mathrm{CL}_{10}\right)$ é de $4,15 \mathrm{ppm}$ e a toxicidade máxima $\left(\mathrm{CL}_{90}\right)$ é de 39,8ppm (Tabela 1).

0 óleo extraído de folhas de Anacardium bumile causa $100 \%$ de mortalidade em larvas de $4^{\circ}$ estádio de Aedes aegypti nas concentrações até $0,125 \%$, o que parece indicar que os ingredientes ativos estão na fase mais apolar. A concentração letal $\left(\mathrm{CL}_{50}\right)$ para o óleo de Anacardium bumile, dosagem que deverá ser aplicada nos testes de campo, é de 20,9ppm.

Tabela 1 - Atividade larvicida do óleo de Anacardium humile sobre larvas de terceiro estádio de Aedes aegypti.

\begin{tabular}{ccc} 
CL10 (IC 95\%) ppm & CL $_{\text {90 }}$ (IC 95\%) ppm & CL $_{\text {g }}$ (IC 95\%) ppm \\
$4,17(2,15-6,19)$ & $20,9(18,88-22,92)$ & $39,8(37,78-41,82)$ \\
\hline
\end{tabular}

CL: concentração letal, IC 95\%: intervalo de confiança a 95\% de probabilidade, ppm: partes por milhão

\section{DISCUSSÃo}

O óleo de Anacardium humile sobre larvas do Aedes aegypti, em diferentes concentrações $(0,1 \%$ a $0,0125 \%)$, demonstrou elevada toxicidade. Resultados semelhantes foram observados em estudos realizados em espécies do gênero Anacardium ${ }^{13}$, onde foi relatado o efeito larvicida que o óleo de Anacardium occidentale apresentou sobre larvas de Aedes aegypti. Estes resultados comprovam o potencial inseticida que plantas do gênero possuem, podendo se tornar uma alternativa para 0 controle deste inseto.
No entanto, a alta toxicidade pode ser considerada um elemento favorável, uma vez que pode ser associada a produtos químicos que perderam a potencialidade pelo uso recorrente e agir como coadjuvante. É necessário, entretanto, testar o efeito residual das substâncias em condições de campo e determinar a possibilidade da utilização segura ao ambiente.

Em relatos sobre a eficiência do extrato das flores da Anacardiaceae Lannea velutina A. Rich no controle de larvas de Anopheles gambiae, Culex quinquefasciatus e Aedes aegypti comprovou-se atividade larvicida ${ }^{7}$. E ainda ensaios com o extrato etanólico das partes aéreas de Anacardium occidentales sobre as larvas de Aedes aegypti, foi definido o perfil de toxicidade, $\mathrm{CL}_{50}$ e a mortalidade total ${ }^{13}$.

No presente experimento, ficou evidente que as frações de diferentes polaridades obtidas das folhas de Anacardium bumile foram inativas sobre as larvas de Aedes aegypti. Assim, novos estudos devem ser conduzidos utilizando outros órgãos vegetais da espécie em questão, bem como, outros métodos e solventes utilizados na extração.

Outro aspecto a ser considerado refere-se à complexidade da composição química dos produtos vegetais, os quais geralmente não causam deposição no ambiente. Além disso, a concentração determinada neste estudo pode ser considerada relativamente baixa, diminuído a possibilidade de aparecimento de populações resistentes.

Os resultados com o óleo das folhas de Anacardium bumile são promissores na busca de larvicidas naturais. No entanto, pesquisas adicionais são necessárias, para proporcionar 0 uso seguro deste produto como princípio farmacológico, uma vez que a determinação da composição química já está sendo executada.

\section{AGRADECIMENTOS}

Ao Ministério de Ciência e Tecnologia (MCT), Centro de Pesquisas do Pantanal (CPP), Conselho Nacional de Desenvolvimento Científico e Tecnológico (CNPq) e a Fundação de Apoio ao Desenvolvimento do Ensino, Ciência e Tecnologia do Estado do Mato Grosso do Sul (FUNDECT) pelo apoio financeiro.

\section{REFERÊNCIAS}

1. Barreto CF, Cavasin GM, Silva HHG, Silva IG. Estudo das alterações morfohistológicas em larvas de Aedes aegypti submetidas ao extrato bruto etanólico de Sapindus saponaria Lin (Sapindaceae). Revista de Patologia Tropical 35:37-57, 2006.

2. Blumer, L. The bioassay part of this study is base don a protocolor development by K. Winnett-Murray, 1997. Proceedings of the 18th Workshop/conference of the Association for Biology Laboratory Education (ABLE), p.249 -271, 2005.

3. Carvalho MP, Santana DG, Ranal MA. Emergência de plântulas de Anacardium bumile A. St.-Hil. (Anacardiaceae) avaliada por meio de amostras pequenas. Revista Brasileira de Botânica 28:627-633, 2005.

4. Cavalcanti ESB, Morais SM, Lima MA, Santana EWP. Larvicidal Activity of Essential Oils from Brazilian Plants against Aedes aegypti L. Memórias do Instituto Oswaldo Cruz 99:541-544, 2004. 
5. Consoli RA, Mendes NM, Pereira JP, Santos BS, Lamounier MA. Influência de diversos derivados de vegetais na sobrevida das larvas de Aedes fluviatilis (Lutz) (Diptera: Culicidae) em laboratório. Memórias do Instituto Oswaldo Cruz 83: 87-93, 1989 .

6. Correia SJ, David JP, David MJ. Metabólitos secundários de espécies de Anacardiaceae. Química Nova 29:1287-1300, 2006.

7. Diallo D, Marston A, Terreaux C, Toure Y, Paulsen BS, Hostettmann K. Screening of Malaian Medicinal plants of antifungural, larvicidal, mollucicidal, antioxidant and antiradical scavenging activities. Phytotherapy Research 15:401-406, 2001.

8. Furtado RF, Lima MGA, Andrade Neto M, Bezerra, JNS, Silva MG. Atividade larvicida de Óleos Essenciais Contra Aedes aegypti L. (Diptera: Culicidae). Neotropical Entomology 34: 843-847, 2005.

9. Gonçalves JLS, Lopes RC, Oliveira DB, Costa SS, Miranda MMFS, Romanos MTV, Santos NOS, Wigg MD. In vitro anti-rotavirus activity of some medicinal plants used in Brazil against diarrhea. Journal Ethnopharmacol 99:403-407, 2005.

10. Lameira CP, Coelho GLV, Mothé CG. Extração de lipídeos da amêndoa de castanha de caju com supercrítico. Ciência de Tecnologia de Alimentos 17:405-407, 1997.

11. Lans C, Hapert T, Georges K, Bridgewater E. Medicinal plants used for dogs in Trindad and Tobago. Preventive Veterinary Medicine 45:533-537, 1999.
12. Lima EP, Oliveira Filho AM, Lima JWO, Cavalcanti LPG, Ramos Junior AN, Pontes RJS. Resistência do Aedes aegypti ao Temefós em Municípios do Estado do Ceará. Revista da Sociedade Brasileira de Medicina Tropical 39:259-263, 2006.

13. Mendonça FAC, Silva KFS, Santos KK, Ribeiro Júnior KAL, Santana AEG. Activities of some Brazilian plants against larvae of the mosquito Aedes aegypti. Fitoterapia 76:599-798, 2005.

14. Pott A, Pott VJ. Plantas do Pantanal. Empresa Brasileira de Pesquisa Agropecuária, Brasília, p. 320, 1994.

15. Reinehr CO, Soares LAA. Lipídios em nozes, Castanha de Caju e Castanha-do-Pará Revista de Ciências Exatas e Engenharias 12:35-45, 2002.

16. Sant'Anna-Santos BF, Thadeo M, Meira RMSA, Ascensão L. Anatomy and histochemistry of stem secretory structures of Spondias dulcis Forst. F. (Anacardiaceae). Revista Árvore 30: 481-489, 2006.

17. Silva HHG, Geris R, Rodrigues Filho E, Rocha C, Silva IG. Diterpenoids from Copaifera reticulata ducke with larvicidal activity against Aedes aegypti (L.) (Diptera, Culicidae). Revista do Instituto de Medicina Tropical de São Paulo 50:25-28, 2008.

18. Simas NK, Lima EC, Conceição SR, Kuster RM, Oliveira Filho AM. Produtos naturais para o controle da transmissão da dengue - atividade larvicida de Myroxylon balsamum (óleo vermelho) e de terpenóides e fenilpropanóides. Química Nova 27:46-49, 2004. 